\title{
Information Society Technologies in Healthcare
}

\author{
Dimitrios G. Katehakis ${ }^{1}$, Manolis Tsiknakis ${ }^{1}$, and Stelios C. Orphanoudakis ${ }^{1,2}$ \\ 1 Center of Medical Informatics and Health Telematics Applications (CMI-HTA) \\ Institute of Computer Science, \\ Foundation for Research and Technology - Hellas (FORTH) \\ P.O. Box 1385, GR-71110 Heraklion Crete, Greece \\ \{katehaki, tsiknaki, orphanou\}@ics.forth.gr \\ 2 Department of Computer Science, University of Crete, \\ P.O. Box 2208, GR-71110, Heraklion, Crete, Greece
}

\begin{abstract}
The growing demand for more efficient and effective healthcare services, coupled with an implicit requirement for supporting citizen mobility and continuity of care, is currently setting the stage for the exploitation of Information and Telecommunications Technologies in the health sector. The current vision comprises affordable wireless access to healthcare resources and services for all citizens, thus making medical expertise a shared resource wherever and whenever needed. Important areas in which Information Society Technologies are likely to have a significant impact include those of pre-hospital health emergencies, remote monitoring of patients with chronic conditions, and medical collaboration through sharing of health-related information resources. Accessibility to these and other media-rich, user-oriented services, in the context of the emerging Global Information Society, will be supported by a Healthcare Information Infrastructure (HII), which can achieve effective horizontal integration of networked information sources.
\end{abstract}

\section{Introduction}

The great challenge in applying information and telecommunications technologies in the health sector is how to be creative in exploiting the opportunities afforded by these emerging technologies, so that peoples' lives may be improved. At the same time, clinically significant and cost-effective, added-value services to the healthcare community ought to be provided, and the potential benefit to be derived from technological advances must also find its way to the scene of an accident, the (virtual) home, and the (virtual) working place of all citizens.

The health sector can serve as the test-bed for assessing the impact which new information and telecommunication technologies will have on our lives. For the past several years, we have witnessed revolutionary technological developments, which afford us a pragmatic look into our digital future. However, the stringent requirements for Quality of Service (QoS) will make the process of change in the health sector evolutionary rather than revolutionary. A plethora of unresolved technical, economic, regulatory, legal, and training issues would have to be settled 
before the real impact of technological change in this important sector can be fully assessed.

Priorities for the 21st century include a shift toward citizen-centered services, meeting QoS requirements, and a substantially greater emphasis on wellness and prevention. Healthcare professionals will continue to deliver care, but will also be increasingly required to share their knowledge and expertise with other colleagues and citizens in general. The new paradigm provides that informed citizens care for their own health and various cooperating stakeholders, who may also be responsible for the continuity of health services within a region, have an impact on the operation of the healthcare system as a whole. In this context, decentralized healthcare can be supported by integrated services for seamless and personalized information delivery, while services and information must be accessible without (visible) organizational boundaries. Information and Communication Technologies (ICT) support information distribution and sharing for health promotion, pre-hospital health emergency medicine, primary health care, hospital services, rehabilitation, home care and other relevant health services.

The Health Telematics Services that are currently being developed and are partially in use should address the needs of all involved actors. Potential users of such services are: (a) health administrators, and the regional and national health authorities, (b) healthcare professionals, and (c) patients and citizens at large. Specifically,

- For health administrators and the regional and national health authorities, continuity of care results in a reduced number of repeated exams, better monitoring and management of healthcare procedures, and better use of available technical and financial resources.

- For healthcare professionals, the secure exchange of medical information, for teleconsultation purposes, efficient access to a patient's distributed Electronic Health Record, and the requirement for medical second opinion or updated training, increase the demand for continuing education, and necessitate the design and implementation of increasingly sophisticated information access and management tools.

- For patients and citizens in general, one must provide access to information and services anywhere and anytime, while supporting mobility and continuity of care.

Thus, a key challenge facing researchers and technology providers is to provide a technological and organizational framework that can integrate a heterogeneous collection of healthcare resources. This framework will increase information availability and ought to address the demanding information processing requirements of healthcare actors.

\section{Application Domain}

The healthcare domain is characterized by a hierarchical structure and involves a large number of specialties with different skills and requirements. The geographical distribution of resources is non-uniform and in many cases these are 
located in remote and isolated areas. Actors in the healthcare domain possess diverse profiles and often lack adequate education and training in the use of technology. In addition, the large volume of available information is inherently heterogeneous and multimedia in nature, while new information is produced at a very high rate. Finally, paper-based health records and other official documents are frequently illegible, scattered, and largely inaccessible. This makes routine sharing of information and data collection very inefficient, which in turn has an adverse effect on the quality of care.

The primary goal of healthcare networking is to provide support for remote expert consultation based on locally acquired medical data and remote guidance for locally performed medical procedures. Integrated healthcare networks support a set of added-value telematic services, implemented over an advanced telecommunications infrastructure, and supported by different information technologies and related applications. The main goal is to provide different levels of support for remote monitoring, as well as preventive, diagnostic and therapeutic medical procedures. Thus, an integrated healthcare network can be considered as an extended virtual healthcare institution that encompasses available physical and human resources over a wide region, in order to support remote medical procedures and patient management.

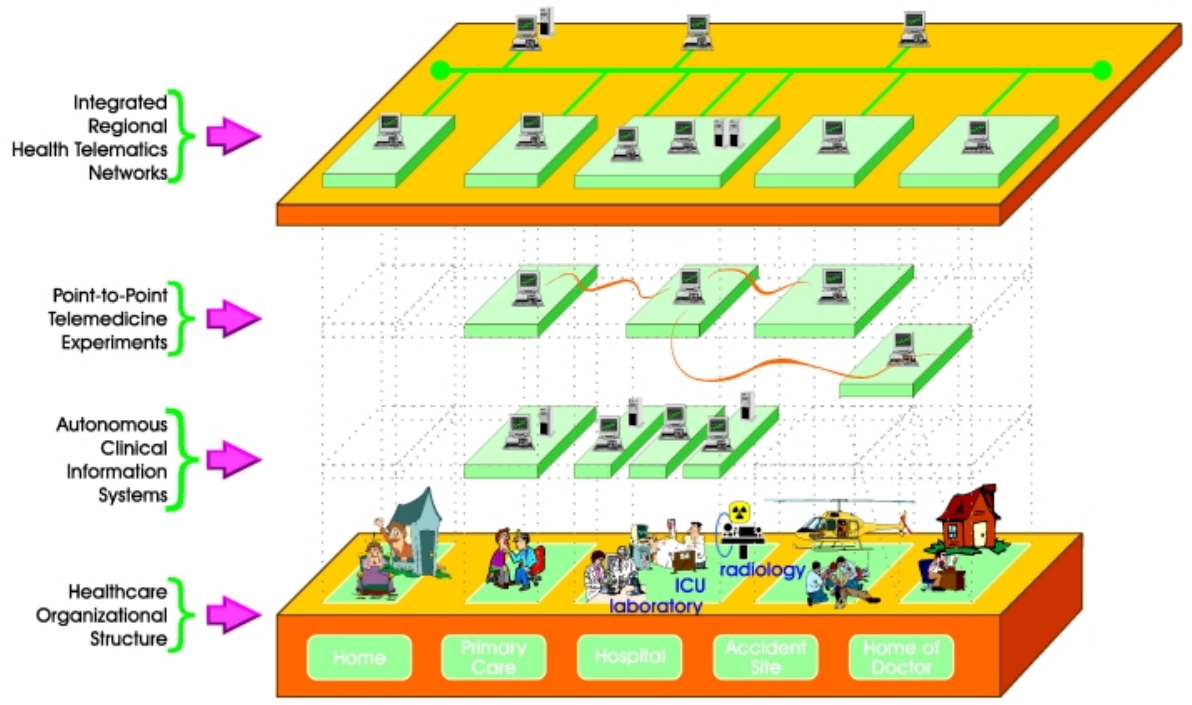

Fig. 1. Evolution of the Healthcare Networking Environment (Starting at the Bottom and Moving to the Top)

The four main application areas of telematics in healthcare are: home care, primary care, hospital care, and pre-hospital health emergency medicine. The traditional environment of autonomous clinical information systems, with point- 
to-point communication used to conduct telemedicine experiments, results in fragmentation of information and cannot be used to fully exploit the potential of the underlying technologies. Integrated regional health-telematics networks (Fig. 1) are currently at advanced stages of development and aim to provide round the clock services to the citizen, not limited by resource availability constraints.

A fundamental prerequisite for the establishment of a scaleable regional health telematics network is the development of an architecture and tools for the integration of specialized autonomous applications that are supported by a HII. Based on an effective horizontal integration of networked information services, the interoperability of applications and services within a health care institution, the interconnection of different institutions, and the intelligent management of health related information within such an integrated network, can be supported.

\subsection{Primary Care}

Primary care is usually offered by General Practitioners (GPs) in primary healthcare centers, as well as by community doctors. Its mission is to provide primary medical and hospital care, preventive medicine, health promotion and social care. Primary healthcare professionals require the means to manage clinical information in a way which is tailored to their needs.

Storing patient encounter information and examination results are typical uses of primary care clinical information systems. Additional functionalities include the direct acquisition of laboratory and imaging examination results, so that they can be seamlessly integrated into personal health records and be readily available on demand.

\subsection{Hospital Care}

A number of clinical departments, offering complementary services, cooperate to provide care at a hospital level. Each department has its own needs for keeping health records and for communicating with cooperating departments (both inpatient and outpatient). In a typical information flow scenario within a hospital, a medical or paramedical professional in a requesting department of a hospital (e.g. pathology) may request the examinations (e.g. biochemical, imaging) of certain patients who are being cared for in the clinic. The different clinical information system in which these examinations are stored must then communicate and possibly exchange information in order to respond to such a request.

The communication and sharing of information among autonomous systems with different data (and event) requirements may involve extensions to these systems. The implementation of such extensions may require the agreement of respective vendors, and certainly increases the cost of development. Therefore, interoperability of systems and services, based on relevant standards, ensures the prompt propagation of information and the efficient use (re-use) of software to order medical examinations, access examination results, and manage workflow. 


\subsection{Home Care}

Home care is intended for the remote monitoring of patients with chronic conditions. Typically, healthcare professionals and patients or the elderly at home communicate with each other via videoconferencing. High-speed broadband videoconferencing sessions and high-quality signal transmission between specialized medical instruments (e. g. stethoscope, spirometer) may also be required. In these cases, bandwidth management becomes particularly important.

In certain clinical applications, such as dermatology, it may also be necessary for the healthcare professional to remotely control the pan-tilt motion of the camera viewing the patient at home. Biosignals acquired at the patient's home are transmitted and displayed for viewing on the healthcare professional's monitor.

The monitoring and follow-up of patients at home is currently finding extensive use in cases when, for various reasons, patients cannot be transported to a GP's office or a hospital or in order to reduce costs. In the future, home care activities will be extended to the virtual home and workplace of an increasingly mobile population, as mobility issues are resolved and the "anyone, anywhere, anytime" paradigm becomes a reality.

\subsection{Pre-hospital Health Emergency Medicine}

The primary tasks of health emergency are: (i) to provide high quality health care services, to the extent possible, at the point of need and (ii) to transfer the victims of accidents and those in some other critical condition to an appropriate healthcare facility. In both cases, it is absolutely essential that the response time is minimized and that the accident victim or patient's condition is monitored closely during transfer to a healthcare facility. Health Emergency Coordination Centers (HECCs) have unique emergency telephone numbers, and need to have access to accurate information regarding the location of an emergency episode, the positioning of ambulances and other mobile units, the qualification of the staff on board, and the availability of healthcare facilities and other resources in the region.

The functional blocks of a health emergency management system are shown on Fig. 2. These include the direct communication of multimedia medical data from mobile units to the HECC, mobile unit tracking and route guidance, resource management at a regional level, and the management of the EHR component related to a specific emergency episode.

Current emergency management systems operate in a sub optimal manner due to the lack or the inadequacy of communication links among the different actors involved. The real issue is not the lack of information and telecommunications technologies, but rather the lack of integration among the different information sources and communication networks. The most important building blocks of such an integrated information infrastructure are the local and the wide are networks (terrestrial, wireless, and mobile), the user terminals for the mobile units, and information systems for the HECC and the positioning of mobile resources. 


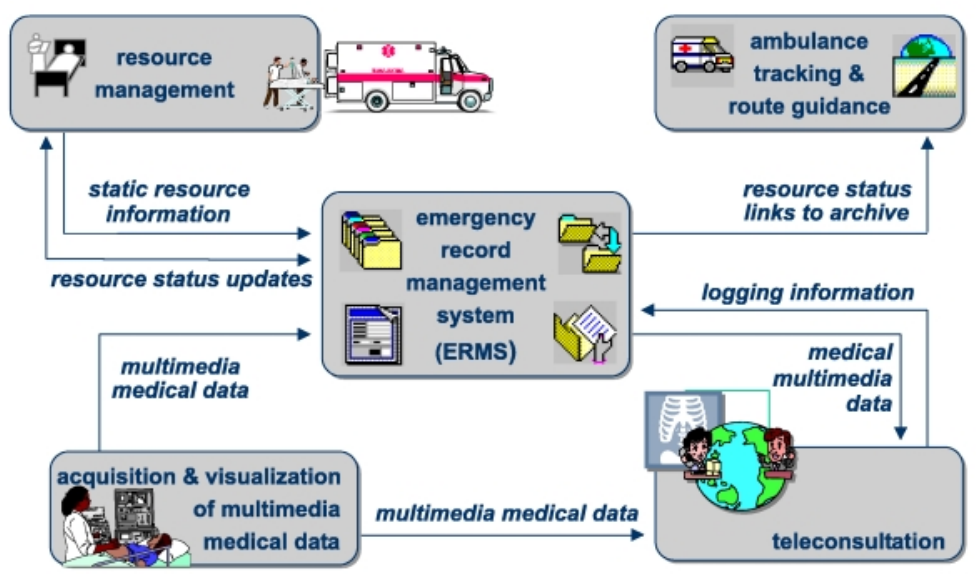

Fig. 2. A Pre-Hospital Health Emergency Management System

\section{$3 \quad$ Health Telematics}

Health telematics involves the use of information processing tools and telecommunications to provide the functional and operational framework that allows computers to transfer medical data to one another. Telemedicine, a related term, refers to the use of health-telematics in the remote delivery of healthcare services.

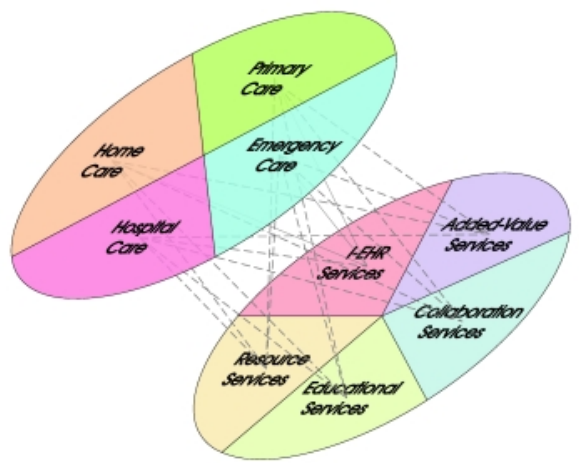

Fig. 3. Healthcare Application Areas and the Five Clusters of Telematic Services

A regional healthcare network supporting integrated user-oriented telematic services to user groups with different needs for information access and QoS requirements presents a significant degree of complexity. This is partly due to the wide spectrum of services that need to be provided and of the requirement for 
interoperability of telematic services and stand-alone applications. Furthermore, user-oriented telematic services need to be offered in a collaboration environment that ensures the continuity of care and information sharing, under strict security and authorization policies.

The telematic services, which are typically delivered within a regional healthcare network, may be classified into five basic classes: collaboration, resource, educational, integrated electronic health record (I-EHR), and added-value. These classes of telematic services are applicable to all four healthcare application areas of home, primary, hospital, and emergency care (Fig. 3).

Collaboration services bridge the gap created by the physical distance separating the various users, while promoting social interaction and the exchange of vital information. This type of user-oriented, telematic services includes teleconsultation, and tele-monitoring. Tele-consultation sessions, among healthcare professionals, may compensate for the lack of experienced or specialized personnel at remote sites, at the site of an accident, or at primary care facilities. They are intended to address emergencies or to evaluate the severity of a case. Tele-monitoring services support patient-physician or physician-physician collaboration for the purpose of delivering healthcare at the home of the patient or monitoring (remotely) a medical examination. Furthermore, as far as community care is concerned, collaboration among user groups that share the same chronic medical condition may provide comfort through the sharing of useful information and experience (special interest groups).

Resource services provide information on the availability of physical resources such as hospital departments, diagnostic modalities, and mobile emergency units, and their characteristics through regional white and yellow page directories. Various sectors of the population and isolated communities benefit from the availability of resource services over the World Wide Web (WWW). In recent years, the Internet-based web technology has stimulated the creation of many such yellow pages by hospitals, health authorities etc., making it possible to get practical information about healthcare providers.

Educational services are especially important for rural regions, where access to information sources is limited. In order to raise public awareness, and to effectively support the continuity of care, healthcare professionals must have access to educational material contained in digital medical libraries. Similarly, the public should have access to information relevant to diseases common in their region. Geographic isolation should not hinder the sharing of knowledge among healthcare professionals, who may improve their practice through interaction with repositories of medical cases and other educational material.

An innovative approach to distance learning, which is based on computerbased training and relies on modern methodologies and WWW technologies, is Web Based Training (WBT) [1]. WBT provides a virtual environment for distance learning by anyone, at any geographic location, and anytime. In a WBT environment, web browsers are used to support 3D reality, video conferencing, chat and other interactive discussion media. Thus, users are able to exchange 
messages with their tutors and among themselves, while bulletin boards and discussion lists facilitate rich electronic interaction.

I-EHR services support single-point access to clinical information by both patients and physicians. They provide a uniform view of data, which may be configured differently at different locations. This is necessary due to the fact that segments of a patient's electronic health record may be found at different healthcare facilities visited by the patient.

I-EHR services require the existence of an underlying technological infrastructure, in order to facilitate the sharing of patient clinical information. Technological challenges are primarily related to information access and storage. Other problems, not necessarily related to technology, include patient identification, as well as making systems inter-operate not only at the control level, but also at the semantic level. Since confidentiality concerning role-based access to information is important, a number of medico-legal issues remain to be resolved, so that access to the EHR data by authorized healthcare professionals in different institutions may be facilitated. The requirement for shared care and continuity of care makes I-EHR services particularly important.

Finally, added-value services extend all the above services and provide specialized support to healthcare professionals. Image processing services, access to specialized indexing and search engines, information filtering and pre-fetching, content-based image indexing and retrieval, and decision support are examples of services that facilitate efficient and effective utilization of the information space.

\section{Information Society Technologies}

Although relational databases are currently used extensively for storing enterprise data, the Internet is becoming the means for integrating systems. In addition, current advances in wireless technology will soon offer the possibility of creating a telemedicine infrastructure for the wireless world. Consequently, next generation clinical information systems will be capable of managing the distributed segments of the EHR and will be based on technologies that support mobility and provide intelligent and personalized access to information sources.

Technologies for the integration of EHR related information mainly involve directories for creating distributed, hierarchical structures of accessible resources, distributed object computing to implement advanced modular functionalities, Internet and Java to glue pieces of information scattered throughout the world, portable devices and mobile communications to enable access from anywhere at any time, and Human Computer Interaction (HCI) technologies to support universal access.

Directories are specialized databases with a hierarchical information model, which are defined in terms of open, standardized, and vendor-neutral protocols. Important strengths of directory technology are its distributed nature and fast lookup capabilities. In essence, the directory is a distributed database capable of storing, in a hierarchical data model, information about people and objects at various servers or network nodes. It is these servers, which provide the poten- 
tially global access to information, made possible by X.500 [2]. Current directory technologies use the Internet standards, conform to a global naming structure, provide public interfaces for directory access (e. g. Lightweight Directory Access Protocol-LDAP), integrate and maintain synchronization with other transitional directories, and support security.

Unlike client/server architectures, where the client discovers and communicates directly with the server, when dealing with distributed object computing, communication middleware acts as an extra functional layer between clients and servers. This additional layer allows applications to be developed without knowledge of the location or any given implementation of all external functionalities. The main examples of generic approaches to middleware today are the Common Object Request Broker Architecture (CORBA), standardized by the Object Management Group (OMG) [3], and Microsoft's Distributed Common Object Model (DCOM) [4]. Distributed object middleware services include communications, naming and directory services, object life cycle management, security and authentication, notification service, and various development and debugging tools.

The main advantages offered by CORBA include architecture, language and location independence. Furthermore, naming and trading services are also available to act as white and yellow pages respectively. DCOM, like CORBA, adheres to separation of interface from implementation using Microsoft's Interface Definition Language (IDL), which is not compatible with CORBA IDLs. Like CORBA, DCOM provides both static and dynamic bindings for methods, and its interface is language neutral. DCOM objects, also known as ActiveX objects, implement all the interfaces the object supports. Java is a programming language similar to $\mathrm{C}++$, easy to learn, efficient, secure, and portable. It produces very compact runtime, which can be used to implement executable content on the web [5]. During the past few years, it has received tremendous support from industry and, consequently, a number of supporting technologies, such as JDBC, JavaBeans, JavaBlend, and Enterprise Java Beans, have been developed.

Recently, the Extended Markup Language (XML) has attracted great attention and is becoming the preferred language for data interchange over the WWW. Requirements best addressed by XML include customizing front-end applications, data mining searches, synthesis of multiple information sources, and electronic commerce-type applications [6]. The Extensible Style Language (XSL), which is a map language that allows transforming XML documents into other language documents, further enables the seamless presentation, and personalization of information.

Research work in the field of HCI focuses on the design of User Interfaces (UIs) accessible and usable by different user groups and the propagation of existing guidelines into user-adaptable UI implementations [7]. This work has been driven by the ever-increasing number of computer users, characterized by diverse abilities, skills, requirements, and preferences, as well as the proliferation of technological platforms, giving rise to a variety in contexts of use, thus necessitating the development of self-adapting user interfaces. 
The combination of fully distributed, n-tier architecture technologies, together with XML, can support the creation of virtual services and secure access to a diverse set of services, in a single integrated environment. In addition, they can efficiently manage network elements and systems using legacy protocols. In all cases, end users need only access Internet, while all implemented underlying functionalities of the integrated network remain fully transparent to them (Fig. 4). What lies ahead is Internet and wireless technologies: the mobile network evolution has been moving from general packet radio services of 1999, to the GSM evolution of 2000, and the 3rd Generation Mobile System (Universal Mobile Telecommunications System — UMTS) of 2001, continuously enhancing multimedia capabilities, internet access and support for IP-based services [8]. This will allow in the future for service portability and seamless roaming of packet, data based services, and bandwidth on demand.

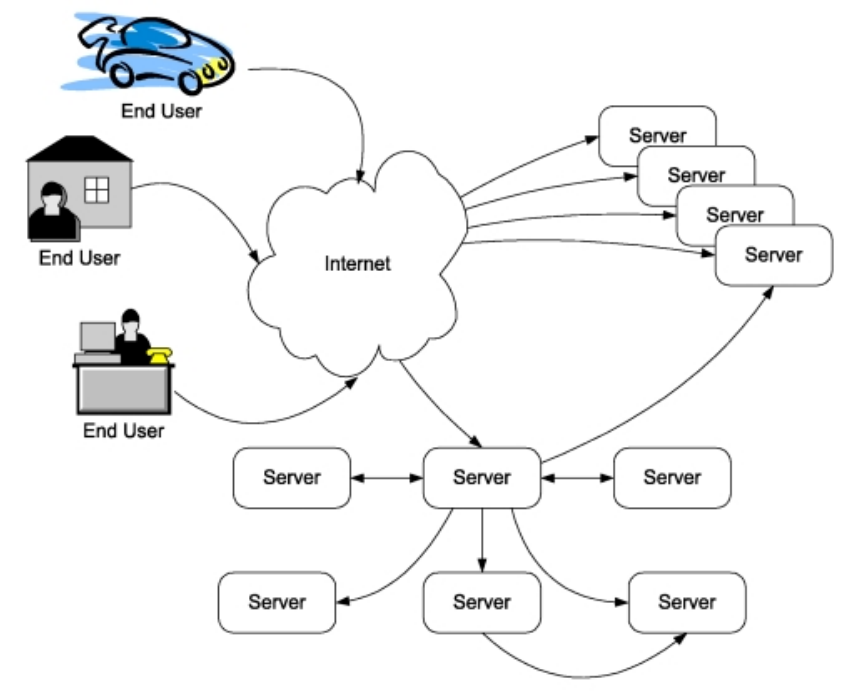

Fig. 4. Schematic Representation of an n-Tier Architecture Over the Internet, Facilitating the Existence of Virtual Environments (home, working place, etc.)

Gradually, more and more devices are being equipped with more processing power and wireless communications capabilities, such as the Infrared Data Association (IrDA) and Bluetooth [9], which allow users to make effortless, wireless and instant connections between various communication devices. This enables more and more appliances to be networked and interoperate, creating new possibilities for home automation and active environment services. 


\section{Related Work}

Work related to the development of a HII falls in two main categories; the first one is associated with standardization activities by international standardization bodies, while the second deals with conscious efforts, in the form of projects or initiatives, to establish a framework for integrating heterogeneous sources of information into a seamless network of health telematics services.

There are different reasons why standards are needed in the healthcare domain. One such reason is that standards allow computer documentation to be consistent with paper-based medical records. Another reason is that information sharing (communication) among different actors, for the purpose of addressing an end-user's problem, is facilitated by the existence of standards-based integrated environments. This includes all agreements on data and context that needs to be shared, so that decision support is provided and there is a return on investment. Healthcare domain standards can be distinguished into standards for terminology, data communication, and software component interoperability.

Medical informatics terminology standards provide for coding schemes and concept representations. These include the READ codes that describe patient care and treatment [10], Laboratory Observation Identifier Names and Codes (LOINC) [11], the Systematized Nomenclature of Medicine (SNOMED) [12], the International Classification of Diseases, Ninth Revision, Clinical Modification (ICD-9-CM), which is based on the World Health Organization's Ninth Revision, International Classification of Diseases (ICD-9) [13], the International Classification of Diseases, Revision Ten (ICD-10) [14], the International Classification of Primary Care (ICPC) [15], and the National Library of Medicine's (NLM) controlled vocabulary thesaurus, known as Medical Subject Headings (MeSH) [16].

Medical informatics data communication standards include those by Health Level Seven (HL7) for the exchange of healthcare related information [17], $\mathrm{X} 12 \mathrm{~N}$ for insurance and insurance-related business processes [18], Digital Imaging and Communications in Medicine (DICOM) [19], as well as those by the National Council for Prescription Drug Programs (NCPDP) [20], the Institute of Electrical and Electronics Engineers (IEEE) [21], the American Dental Association (ADA) [22], the American Nurses Association (ANA) [23], and the European Standardization Organization CEN/TC251 [24].

The only effort so far at establishing standardized software component interoperability, comes from CORBAmed, the Healthcare Domain Task Force (DTF) of the OMG [3]. Standardized object-oriented interfaces, expressed in the Interface Definition Language (IDL), associated with the integration framework of CORBA, that have been adopted so far include services for person identification, accessing clinical observations and imaging, resource access decision, and terminology query. A similar effort by the recently introduced Microsoft Health Users Group [25] is making its initial steps, without having produced any specifications so far. Both types of standards, when utilized, are expected to be particularly important in large hospitals and regional networks, where many kinds of different computers have been installed and cannot be replaced. 
Various research and development projects attempt to provide solutions to technical problems associated with the development of a HII. Central element in all these efforts is the Integrated Electronic Health Record (I-EHR), which is the cornerstone of shared care and the point of reference for any exchange of medical information. Currently, three approaches to the I-EHR exist [26]. The most widespread approach relies on clinical messages to enable the exchange of information among information systems (e.g. HL7), but it does not scale well when the number of possible system interactions increases. Clinical data warehouses offer an alternative way for integrating information, but when used in practice, keeping information up-to-date becomes a real issue [27]. The third approach, that of a federated I-EHR, is based on a common normalized model to map underlying schemas into the overall architecture. Related efforts include that of the STAR [28], GEHR [29], SYNAPSES [30] projects, funded by the European Commission, as well as CEN/TC251 [17], and TeleMed [31] in the USA.

In relation to terminology services, the Unified Medical Language System (UMLS) project [32] of the NLM develops machine-readable 'Knowledge Sources' that can be used by a variety of application programs. This overcomes retrieval problems caused by differences in terminology and by the proliferation of information sources. In the context of the reference architecture, terminology services offered by terminology servers supporting evolving standards will help healthcare professionals and researchers retrieve and integrate electronic biomedical information from a variety of sources.

The real problem with existing standards and recommendations arising from projects/initiatives is that there are many of them, they are difficult to adopt, and they are always in a state of development. Nevertheless, one does not have to wait for standards to be fully developed before attempting to exploit their potential benefits.

\section{Architectural Framework}

Organizational and procedural context ought to be taken into account when specifying and developing information systems for the healthcare domain. Fundamental principles in developing any architectural framework include the promotion of interoperability, so that development and maintenance costs are kept low, and the use of open standards, so that the potential for compatibility with the rest of the world is sustained. The implementation of scaleable and extensible modular architectures and the provision of high quality services to society is a desirable end result. In order to achieve this result, it is particularly important that the installed infrastructure can continue to evolve and that, as technologies mature, new and improved capabilities can be taken advantage of. 


\subsection{HYGEIAnet: The Integrated Health Telematics Network of Crete}

The development and operation of HYGEIAnet on Crete (Fig. 5) constitute a conscious effort to provide an integrated environment for healthcare delivery and medical training across the island. HYGEIAnet takes advantage of the increasing capacity of terrestrial and mobile communication networks and the development of advanced telemedicine services to provide every citizen of the island with effective healthcare services and to support remote consultation among healthcare professionals in specialized centers, district and regional hospitals, and other points of care.

In the course of designing and implementing this integrated services network, special efforts are being made to meet the requirements of the user groups involved and to use state-of-the-art technology and standards at every stage. Alternative patient, problem, and case-based architectures for the I-EHR have been considered in an attempt to provide transparent access and secure communication of information between and within medical specialty areas, as well as in a variety of situations from community to hospital care across the region. Various strategies for multi-database integration and interoperability were considered in conjunction with the EHR to provide homogeneous access to its distributed segments at all levels of the healthcare system. Advanced multimedia telematics applications such as telecardiology, telepathology, teleradiology, teleophthalmology, etc., supporting synchronous and asynchronous teleconsultation and cooperative diagnostic work between healthcare providers at different locations are currently being developed and will soon become part of the integrated system.

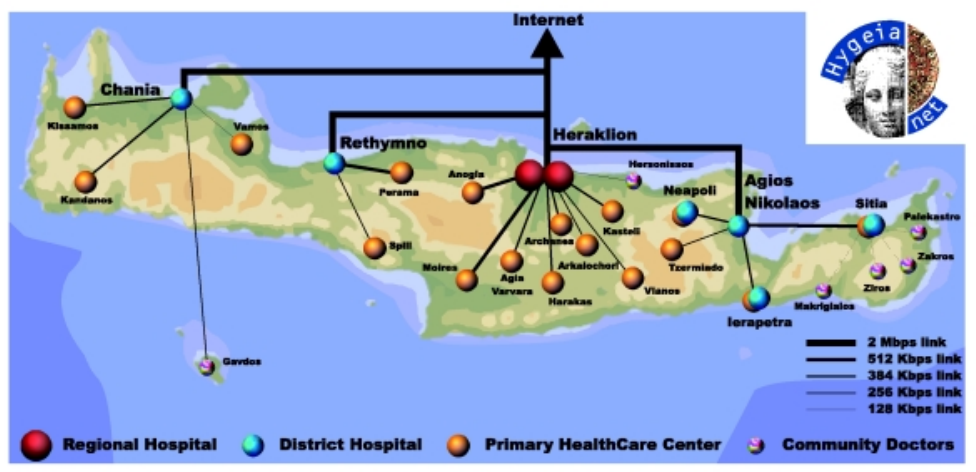

Fig. 5. HYGEIAnet: the Integrated Regional Health Telematics Network of Crete

Short to medium term objectives include the introduction of lifelong electronic health records for all citizens, round-the-clock on-line access to electronic health records and information about best clinical practice, for all authorized 
actors. Currently, HYGEIAnet includes almost the entire public healthcare system of the Region of Crete, which consists of two Regional Hospitals, including the University Hospital, five general hospitals, fifteen primary healthcare centers, and one hundred and seventeen community doctors. HYGEIAnet also includes pre-hospital emergency services provided by the National Center for Emergency Care.

\subsection{HYGEIAnet Reference Architecture}

Information technology planners use the term architecture to refer to abstract technical descriptions of systems. Architectures provide the basic framework for the creation, movement, distribution, use, and management of information [33,34]. In particular, a reference architecture model describes a system in terms of basic functional elements and the interfaces among them. It specifies where protocols need to be defined and identifies groups of functionalities, without imposing a specific physical implementation.

A regional healthcare network, providing integrated user-oriented telematic services to user groups with different information and QoS requirements, presents a significant degree of complexity. This is partly due to the wide spectrum of the services provided. Furthermore, interoperability among telematic services and stand-alone applications is critical, since services and applications share data and information. The reference architecture for the HII, that is shown in Fig. 6, guides the development of a health-telematics network for the provision of integrated services [35]. It conforms to the CEN/TC251 Healthcare Information System Architecture (HISA) [36] and offers several middleware services, which can be used to deploy regional and interregional end-user services. Furthermore, it provides a general framework in which healthcare-related information systems may be integrated to provide media-rich services to healthcare professionals, social workers, and the public. It is important to note that the HII provides a conceptual roadmap, since it does not impose any execution platform, and consists of a vast conglomeration of autonomous information systems and supporting services.

The HII consists of three basic components: applications, enabling (or middleware) services, and network infrastructure. At the bottom, the infrastructure layer provides services that are related to the integration and inter-working of the technological environments. The application layer includes the applications that support user activities in the various areas of an organization. These applications are both information sources and/or information access points. Clinical, diagnostic, and administrative information systems, medical libraries, and user-oriented services are all part of the application layer [36]. All applications and services of the application layer are associated with their own data model and user-interface. In the middleware layer reside generic and healthcare-specific middleware services. Generic middleware services support the applications with general-purpose facilities, which are common to any information system in any type of business domain [36]. Healthcare-specific middleware services support applications with services related to activities of the healthcare domain. 


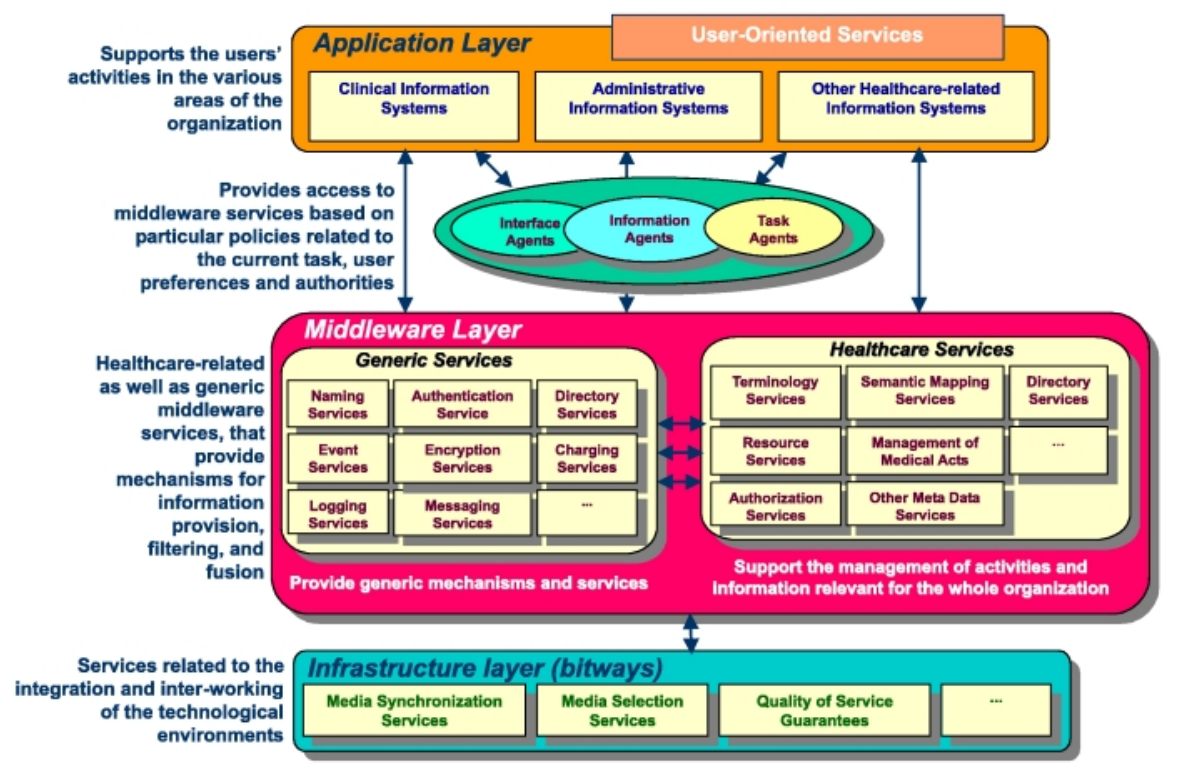

Fig. 6. HYGEIAnet Reference Architecture

System conformance is measured by the capability of an information system to exploit, when necessary, one or more of the common components specified in the reference architecture. The degree of conformance to the reference architecture indicates the capability of the information system to be integrated, from both the functional and information viewpoint, with the rest of the information systems.

\subsection{HYGEIAnet Information Infrastructure}

The core infrastructure components of HYGEIAnet allow for the secure and hierarchically structured information on end-user personalized preferences, the consequent management of all kinds of information resources by means of a Healthcare Resource Directory (HRD) and the Patient Clinical Data Directory (PCDD), a central element of the whole infrastructure. Additionally, the WebOnCOLL collaboration infrastructure allows for the secure creation and management of virtual workspaces and telemedicine session folders.

The organizational structure, medical services, and personnel of the various healthcare facilities comprise the regional X.500 directory of healthcare resources. The directory provides multiple views of the healthcare resources in the region. One view is location-oriented, indexing healthcare resources according to geographic location. Another view follows the healthcare organizational structure, classifying healthcare facilities into tertiary, secondary, and primary ones. Private medical doctors practicing medicine in the region of Crete are also 
included in the directory and classified according to their specialty and location. In the long term, resource services will be maintained by the individual healthcare facilities and accessed through a concentration service maintained by the regional authorities.

The PCDD is a central middleware element of the overall architecture that provides clinical information on the distributed EHR segments maintained by autonomous information systems [37]. Access to detailed information regarding specific healthcare encounters is delivered via role-based authorization privileges and controls. The administration of the healthcare organizations' business rules, for different user-groups, is made via a specially tailored and developed ruleeditor [38]. In its current deployment status (as of August 2000), PCDD offers access to various distributed healthcare sites (medical units) in the region of Crete, and to respective distributed clinical information systems (the legacy feeder systems).

The collaboration infrastructure of $W e b O n C O L L$ is based on virtual workspaces and user profiles [39]. Virtual workspaces implement collections of heterogeneous objects, maintain history data, and support awareness regarding content updates and user interaction. Agents (human users or applications) connected to a workspace may create or add objects into the workspace and are notified of changes in the contents of the workspace or when other agents are connected to the same workspace. User profiles enable the customization of workspaces according to user authorities, tasks, and preferences. User profiles also maintain account information and access information on subscribed services such as personalized information services. The interoperability of WebOnCOLL with enabling middleware services of the regional HII ensures availability of accurate resource information and certification of the exchanged data.

\subsection{Middleware Services and Components}

The initial set of core services supporting the utilization of an I-EHR environment at a regional level, within the framework of the HYGEIAnet Reference Architecture, has been identified and an initial prototype implementation is available providing useful feedback for future enhancements. At present, core services are based on basic infrastructures and include the following:

Authentication services allow access only to authorized end-users. They are implemented by means of a security server that manages the user rights and roles, together with associations to personalized profiles. Its purpose is to certify the role and authority of both users and services (or applications) within the regional healthcare network. In this context, any patient is able to have complete access to all personal information.

Encryption services are responsible for the secure communication of sensitive personal information over the Virtual Private Network (VPN) of HYGEIAnet, as well as the Internet. The combination of digital signatures for authentication, public key cryptography for recipient authentication, and Secure Socket Layer (SSL) protocols for secure data-transfer, provide the 
necessary technological framework for secure communication of healthcare related information across the Internet.

Patient identification services allow for the unique association of distributed patient record segments to a master patient index. This is a very challenging task to perform and one of the major barriers in developing a reliable I-EHR environment. This is because no global, unique person identification exists worldwide today. In addition, even for countries that possess one, this is not used internally for identification purposes by clinical or administrative information systems. Apart from person identifiers, identifiers are also needed for providers, locators, devices, etc.

Auditing services are responsible for recording all performed interactions between middleware services and/or end-user applications, directly or indirectly. The logs produced can then be used for both tracing back transactions in time and for charging. They can also be used as a profiler tool for individual server loads, and for the construction of end-user interest profiles by means of data mining techniques.

Resource location services are useful for identifying available resources and the means for accessing them. Examples of resources include pharmacies onduty, hospitals and clinics, clinical information systems available at a regional level, methods and technologies available for accessing primary information, and protocols for exchanging information with them.

Encounter location services are used for locating primary information found in different patient record segments. In this context, encounter is the term used for describing clinical information that is produced during the communication about the patient, between two or more individuals, at least one of whom is a member of the responsible healthcare team.

Clinical Observation Access Services are required for obtaining primary information directly from specific clinical information systems. This service requires the implementation of standardized gateways for each clinical information system for securely importing/exporting patient record data.

User Profile Services track the long-term interests of users and are used for maintaining personalized settings and preferences [40]. User profiles are made up of terms/keywords to describe interesting information, or examples of interesting information, and are utilized for the selective dissemination of information to their owners. User profiles initially are created according to existing stereotypes that are automatically assigned to them, after gathering some initial user-specific information, and users may actively contribute to the incremental building of their profiles.

Terminology Services have a dual objective: to map and associate existing coding schemes, and to relate the internal semantics of the clinical information systems maintaining primary information. The first objective is accomplished by means of UMLS, while the second requires a conscious effort by clinical information systems developers and people responsible for maintaining I-EHR services up to date. 


\subsection{Act Management - Workflow Issues}

Management of acts involves the coordinated execution of multiple tasks performed by different healthcare actors. Important requirements for the efficient and reliable execution of applications supporting workflow management include deep understanding of the processes to be modeled and workflow automation [41]. The separation of work activities into well-defined tasks, rules, and procedures allows for the modeling of healthcare processes in a rigorous and comprehensive manner. Middleware services, associated with the management of medical acts, facilitate functional integration and coordinate medical procedures in terms of quality, throughput, and reliability [42]. The management of medical acts or workflow requires a process definition stage, a workflow processing environment, and tools to support the run-time interaction.

\section{Conclusions}

The implementation and operation of Integrated Regional Healthcare Networks, for the provision of cost-effective and clinically meaningful health telematics services, provide many challenges and opportunities for assessing the impact of Information Society Technologies on our lives. The challenges are not only technological, but are also related to various unresolved medical, legal, regulatory, and economic issues. Furthermore, they are enhanced by the complexity of the healthcare application domain, which arises from the physical distribution of available resources, the heterogeneous user profiles, and the diverse requirements of different levels of care in a regional setting. On the other hand, opportunities for applying information and telecommunications technologies, in this rather sensitive and highly visible application domain, exist precisely because there is a need to meet the above challenges for the purpose of supporting continuity and improved quality of care for an increasingly mobile population. The goal is to develop and manage regional health telematics applications in order to support real-time access to services by anyone, anywhere and anytime. This would also require that adequate bandwidth be allocated on demand across different fixed, wireless, and mobile telecommunications networks.

Information Society Technologies support the integration of systems and services at a regional level and facilitate access to medical information by healthcare professionals and citizens, who are thus empowered to care for their own health. Furthermore, they provide support for evidence-based medical practices and contribute toward improving the efficiency of the health sector as a whole.

The definition and adoption of standards, primarily with respect to the functional interfaces between the various system components, represents a fundamental requirement that must be satisfied before different applications, developed by different suppliers, can be integrated and used effectively in health care. Future breakthroughs in optical communication technology, combined with the availability of more powerful and possibly intelligent mobile terminals, will further support continuity of care and prompt care at the point of need, while making medical expertise a shared resource and enabling seamless access to healthcare 
resources. This will lead to the creation of a virtual distributed healthcare organization. Then, the real challenge will be to make healthcare telematics services accessible by all, at a reasonable cost. At this point, a critical mass needs to be established for the services to become widely available and a conscious effort is required so that a better-informed population can sustain a functioning network of advanced services.

Finally, our duty is to be creative in using the emerging information and telecommunications technologies, so that we may provide support for the delivery of significant and cost effective healthcare services. It is also important that the potential benefit to be derived from technological advances finds its way to the scene of an accident, the (virtual) home, and the (virtual) workplace of all citizens. The adoption of Information Society Technologies in the health sector is a process of change and, therefore, it is likely to be evolutionary rather than revolutionary.

\section{Acknowledgements}

The development of the Regional Health Telematics Network of Crete is a longterm goal of the Center of Medical Informatics and Health Telematics Applications (CMI-HTA), at the Institute of Computer Science of the Foundation for Research and Technology - Hellas (ICS-FORTH). The work reported in this article represents a collaborative effort involving all the members of CMI-HTA, whose significant contributions the authors would like to explicitly acknowledge. This work is being supported in part by several R\&D projects of the European Commission, the General Secretariat for Research and Technology of the Greek Ministry of Development, and the Greek Ministry of the National Economy.

\section{References}

1. Web-Based Training Information Center. http://www.webbasedtraining.com/. 158

2. ITU. Recommendation X.500 - Information technology - Open Systems Interconnection - The directory: Overview of concepts, models, and services, Nov. 1993. 160

3. The Object Management Group. http://www.omg.org/. 160, 162

4. Microsoft COM Technologies. http://www.microsoft.com/com/. 160

5. The Source for Java Technology. http://www.javasoft.com/. 160

6. The World Wide Web Consortium. http://www.w3.org/. 160

7. C. Stephanidis and D. Akoumianakis. Preference-based human factors knowledge repository for designing user interfaces. Int. J. on HCI, 9(3):283-318, 1998. 160

8. M. W. Oliphant. The mobile phone meets the internet. IEEE Spectrum Mag., 36(8):20-28, 1999. 161

9. The Bluetoth Specific Interest Group. http://www.bluetooth.com/. 161

10. The READ Codes. http://www.cams.co.uk/readcode.htm. 162 
11. A. W. Forrey, J. M. Clement, G. DeMoor, S. M. Huff, D. Leavelle, and et al. D. Leland. Logical observation identifier names and codes (LOINC) database: A public use set of codes and names for electronic reporting of clinical laboratory test results. Clin. Chem., 42(1):81-90, 1996. 162

12. Systematized Nomenclature of Medicine. http://www.snomed.org/. 162

13. National Center for Health Statistics: Classification of Diseases. http://www.cdc.gov/nchs/icd9.htm. 162

14. World Health Organization. http://www.who.int/. 162

15. H. Lamberts, M. Wood, and I. M. Hofman-Okkes, editors. The International Classification of Primary Care in the European Community: With a Multi-Language Layer. Oxford University Press, Oxford, 1993. 162

16. MeSH, National Library of Medicine. http://www.nlm.nih.gov/pubs/factsheets/mesh.html. 162

17. Health Level Seven. http://www.hl7.org/. 162, 163

18. X12N. http://www.hipaanet.com/disa_x12n.htm. 162

19. Digital Imaging and Communications in Medicine (DICOM); National Electrical Manufacturers Association. http://www.nema.org. 162

20. National Council for Prescription Drug Programs. http://www.ncpdp.org/. 162

21. The Institute of Electrical and Electronics Engineers. http://www.iee.org/. 162

22. American Dental Association. http://www.ada.org/. 162

23. American Nursing Association. http://nursingworld.org/. 162

24. European Committee for Standardization (CEN), Technical Committee for Health Informatics (TC251). http://www.centc251.org/. 162

25. Microsoft Healthcare Users Group - Europe. http://www.mshug-euro.org/. 162

26. J. Grimson, W. Grimson, and W. Hasselbring. The system integration challenge in health care. Communications of the ACM, 43(6):49-55, 2000. 163

27. W. Hasselbring. Federal integration of replicated information within hospitals. Int. J. Dig. Lib., 1(3):197-208, 1997. 163

28. M. Blazadonakis, V. Moustakis, and G. Charissis. Seamless care in the health region of Crete: The star case study. In MIE 97, pages 157-161, Porto Carras, 1997. http://www.ics.forth.gr/ICS/acti/cmi_hta/publications/papers.html. 163

29. D. Ingram. The good European health record in healthcare in the new communication age. IOS, pages 66-74, 1995. 163

30. J. Grimson. A CORBA-based integration using the synapses approach. Biomedicine, 2(3):124-138, 1998. 163

31. D. G. Kilman and D. W. Forslund. An international collaboratory based on virtual patient records. Com. of the ACM, 40(8):111-117, 1997.

http://www.acl.lanl.gov/TeleMed/Papers/p110-kilman.pdf. 163

32. Unified Medical Language System Documentation, National Library of Medicine. http://www.nlm.nih.gov/research/umls/UMLSDOC.HTML. 163

33. Cross Industry Working Team. An architectural framework for the national information infrastructure. White paper, 1994.

http://www.xiwt.org/documents/ArchFrame.html. 165

34. Cross Industry Working Team. Building the information infrastructure: A progress report, 1999. http://www.xiwt.org/documents/BuildingNII.pdf. 165

35. M. Tsiknakis, C. E. Chronaki, S. Kapidakis, C. Nikolaou, and S. C. Orphanoudakis. An integrated architecture for the provision of health telematic services based on digital library technologies. Intl. J. on DigLibs., 1(3):257-277, 1997. http://www.ics.forth.gr/ICS/acti/cmi_hta/publications/papers/1997/ dglib97/dglib97.html. 165 
36. CEN/TC251/WG1/PT1-013: Medical Informatics: Healthcare Information System Architecture, 1995. 165

37. D. G. Katehakis, P. Lelis, E. Karabela, M. Tsiknakis, and S. C. Orphanoudakis. An environment for the creation of an integrated electronic health record in HYGEIAnet, the regional health telematics network of Crete. In TEPR Procs 1, pages 89-98, 2000.

http://www.ics.forth.gr/ICS/acti/cmi_hta/publications/papers/2000/ tepr2000/tepr2000.html. 167

38. G. Potamias, D. G. Katehakis, E. Karabela, V. Moustakis, and M. Tsiknakis. Role-based access to patients clinical data: The intercare approach in the region of Crete. In MIE, Hannover, 2000. 167

39. C. E. Chronaki, D. G. Katehakis, X. Zabulis, M. Tsiknakis, and S. C. Orphanoudakis. WebOnCOLL: An environment for collaboration in regional healthcare networks. IEEE Transactions on Information Technology in Biomedicine, 1(4):257-269, 1997.

http://www.ics.forth.gr/ICS/acti/cmi_hta/publications/papers/1997/ ieee97weboncoll/ieee97weboncoll.html. 167

40. N. J. Belkin and W. B. Croft. Information filtering and information retrieval: Two sides of the same coin? Communications of the ACM, 35(12), 1992. 168

41. D. Georgakopoulos et al. An overview of workflow management: From process modeling to workflow automation infrastructure. Distributed and Parallel Databases, 3(2):119-153, 1995. 169

42. E. Kaldoudi, M. Zikos, E. Leisch, and S. C. Orphanoudakis. Agent-based workflow processing for functional integration and process re-engineering in the health care domain. In Procs of EuroPACS, pages 247-250, Pisa, 1997. 169 